\title{
openheart Hidden burden of arrhythmias in patients with small atrial septal defects: a nationwide study
}

\author{
Sebastian Udholm, ${ }^{\oplus 1}$ Camilla Nyboe, ${ }^{1}$ Andrew Redington, ${ }^{2}$ \\ Jens Erik Nielsen-Kudsk, ${ }^{3}$ Jens Cosedis Nielsen, ${ }^{3}$ Vibeke Elisabeth Hjortdal ${ }^{\oplus}$
}

\begin{abstract}
- Additional material is published online only. To view please visit the journal online (http://dx.doi.org/10.1136/ openhrt-2019-001056).
\end{abstract}

To cite: Udholm S, Nyboe C, Redington A, et al. Hidden burden of arrhythmias in patients with small atrial septal defects: a nationwide study. Open Heart 2019;6:e001056. doi:10.1136/ openhrt-2019-001056

Received 24 March 2019 Revised 6 May 2019 Accepted 13 June 2019
Check for updates

(c) Author(s) (or their employer(s)) 2019. Re-use permitted under CC BY-NC. No commercial re-use. See rights and permissions. Published by BMJ.

${ }^{1}$ Department of Cardiothoracic and Vascular Surgery, Aarhus University Hospital, Aarhus, Denmark

${ }^{2}$ Pediatric Cardiology, Department of Pediatrics, Cincinnati Children's Hospital, Cincinnati, Ohio, USA

${ }^{3}$ Department of Cardiology, Aarhus University Hospital, Aarhus, Denmark

Correspondence to Dr Sebastian Udholm; sebastian. udholm@clin.au.dk

\section{ABSTRACT}

Background In recent Danish nationwide register-based study, adults with small, unrepaired atrial septal defects (ASD) have increased risk of pneumonia, atrial fibrillation (AF) and stroke. Moreover, they revealed higher mortality than the background population.

Objective In this nationwide study, we evaluate the hidden burden of atrial and ventricular arrhythmias in adult patients with a small, unrepaired ASD without a previous diagnosis of AF.

Methods All Danish patients, aged 18-65, diagnosed between 1953 and 2011 with an unrepaired ASD and no documented AF were invited for 7 days Holter-recording, echocardiography and 6 min walk test. The first 48 hours Holter-recording was completely analysed, while only AF was screened for throughout all 7 days. Furthermore, the entire patient group were characterised using the unique Danish registries.

Results A total of 151 patients (mean age 32 years) were included. Approximately $80 \%$ of the patients had spontaneous closure of their defect. Despite this, occult arrhythmias were frequent. The most common arrhythmia was supraventricular tachycardia $(n=24,16 \%)$ with non-sustained atrial arrhythmias in 21 patients and AF in two patients. A considerable number of patients had non-sustained ventricular tachycardia $(n=12,8 \%)$. Patients with ASD and tachyarrhythmias had increased right ventricular to left ventricular diastolic area in echocardiography and higher age when compared with ASD patients without arrhythmias.

Conclusion Adult patients with small, unrepaired ASD have a hidden burden of both atrial and ventricular tachyarrhythmias. The mechanism likely relates to the residua of previous right-heart volume overload and incomplete reverse remodelling. Our results support guidelines recommending continued follow-up of patients with small, unrepaired ASD.

\section{INTRODUCTION}

Atrial septal defect (ASD) is associated with persistent left-to right shunting and can result in atrial interstitial fibrosis and arrhythmia, right ventricular remodelling, pulmonary hypertension and heart failure in later life. ${ }^{1}$ For this reason, closure in childhood is recommended for those with

\section{Key questions}

What is already known about this subject?

- Patients with a small, unrepaired atrial septal defects (ASD) have an increased risk of atrial fibrillation. The hidden burden of undiagnosed atrial and ventricular arrhythmia prior to the development of symptomatic arrhythmia is, however, unknown.

What does this study add?

- Patients with a small, unrepaired ASD have a hidden burden of atrial and ventricular tachyarrhythmias, especially focal atrial tachycardia and non-sustained ventricular tachycardia, even after spontaneous closure of the defect. Arrhythmias were associated with increased right ventricular to left ventricular area ratio suggesting that associated changes in atrial and ventricular myocardium are partly irreversible.

How might this impact on clinical practice?

- These results support the guidelines for management of adults with small ASD, recommending continued follow-up every 2-3 years with a clinical focus on arrhythmias.

'haemodynamically significant' defects associated with evidence of right-sided chamber dilation. The fate of those in whom the ASD is considered too small to warrant closure and presumably therefore considered to have benign long-term implications, is less well known. Recently, however, these patients, even if spontaneous closure had occurred, were shown to have a higher mortality than the general population, ${ }^{2}$ an average lifespan of 63 years, ${ }^{3}$ and a significant burden of cardiovascular disease with manifestations similar to that of unoperated larger defects. Indeed, while it is well known that adult patients with significant ASD have an increased risk of atrial fibrillation (AF) despite closure of their defect, ${ }^{4}$ interestingly, a similar risk of AF and stroke was reported from registry studies in patients with a small, unrepaired ASD. ${ }^{4}$ While retrospective registry studies are valuable to assess the incidence and effect of overt 
disease, they are generally not designed to assess the prevalence and impact of covert, asymptomatic, disease. We hypothesised that there is a hidden burden of undiagnosed atrial and ventricular arrhythmia in patients with small unoperated ASD prior to the development of symptomatic arrhythmia such as AF. Consequently, we undertook this prospective nationwide cohort study in adult patients with a small, unrepaired ASD without a known diagnosis of AF.

\section{METHODS}

\section{Study design and participants}

In this nationwide descriptive cohort study, we used medical registries in Denmark to characterise patients with small, unrepaired ASD. The cohort was identified in previous studies through the Danish National Patient Registry (DNPR) and the method is thoroughly described elsewhere. ${ }^{45}$ The DNPR includes complete and continuously updated data on all residents living in Denmark since 1977, containing information on all inpatient and outpatient hospital contacts in Denmark, dates of admission and discharge, surgical procedures and discharge diagnoses coded according to the International Classification of Disease (ICD). ${ }^{67}$ Two independent clinicians validated the ASD diagnosis through review of hospital records in adult patients above 18 years of age. Patients with defect closure, persistent foramen ovale, pulmonary arterial hypertension or Eisenmenger syndrome were excluded, thus presumably leaving only small, unrepaired and haemodynamic insignificant ASDs.

All Danish patients diagnosed with an ASD left unrepaired and no previous diagnosis of AF, were invited to a clinical examination at Aarhus University Hospital. The examination included electrocardiography, 7 days Holter-recording, standard echocardiography and $6 \mathrm{~min}$ walking test. Additionally, patients answered questions at inclusion regarding symptoms (specifically signs of arrhythmias), excessive alcohol consumption, whether they would rate their diet as healthy and whether they would rate their general health and physical form as good. Last, the DNPR was used to identify baseline comorbidities from the ICD diagnosis in all included patients. Enrolment was conducted from December 2015 until February 2018. Inclusion criteria were an unrepaired ASD and age between 18 and 65 years. Criteria for excluding subjects were: severe mental or psychiatric disorder incompatible with inclusion, other congenital heart disease (except patent ductus arteriosus), persistent foramen ovale, previous ASD closure and death.

This study was approved by The Danish Data Protection Agency (j.nr. 1-16-02-633-15) and by The Regional Committee on Biomedical Research Ethics of the Central Denmark Region (j.nr. M-2015-197-15).

\section{ECG}

Standard 12-lead ECG was recorded for each patient. We defined first-degree atrioventricular block as a PR interval $>200 \mathrm{~ms}$. The widest QRS complex was determined and a duration $>120$ ms was defined as a complete bundle branch block. Measurements for QRS duration, PR-interval, $\mathrm{P}$ wave duration and $\mathrm{P}$ wave height in II were performed manually by a single observer (SU).

\section{Holter-recording}

A 7-day 3-channel Holter recording (Lifecard CF) was obtained in each patient. For analysing data, we used Spacelabs Healthcare, Pathfinder. The first 48 hours recording was completely analysed, while only $\mathrm{AF}$ was screened for throughout all 7 days. We defined supraventricular tachycardia (SVT) as $\geq 3$ consecutive supraventricular premature complexes and non-sustained ventricular tachycardia (NSVT) as $\geq 3$ consecutive ventricular premature complexes both with a heart rate $>100$ beats/minute for $<30 \mathrm{~s}$. AF was defined as irregular heart rhythm without distinct sinus $p$-waves for $\geq 30 \mathrm{~s}$. Sinus pauses were $>2 \mathrm{~s}$. All findings were validated by a senior electrophysiologist.

\section{Echocardiography}

We used a standardised transthoracic echocardiogram protocol targeting right heart chambers and the atrial septum to determine if defects had spontaneously closed. All tests were performed using a GE Vivid E9 (GE Healthcare, Horten, Norway) by a single highly trained echo-technician. and all images were review by a cardiologist specialised in congenital heart disease.

\section{6 min walk test}

We used the 6 min walk test to assess submaximal exercise capacity. The test was conducted on a flat surface with a straight-lined course. Recorded outcomes were achieved walking distance, oxygen saturation and heart rate.

\section{Statistics}

Continuous, normally distributed data were reported as mean \pm SD. The unpaired Student's t-test was used if normally distributed. Comparisons of categorical baseline characteristics between patients with or without arrhythmias were performed by Fisher's exact tests. Statistical significances were considered with $\mathrm{p}$ values below 0.05 . All analyses were performed using Stata IC 15.1 (Stata, College Station, Texas, USA).

\section{RESULTS \\ Patient characteristics}

The Danish ASD cohort is composed of 2277 validated adult patients with ASD. Of these, 1554 underwent closure either surgically or by catheter, providing a total of 723 patients with an unrepaired ASD. A total of 119 patients with an unrepaired ASD had a known diagnosis of $\mathrm{AF}$ and so were excluded. Approximately half $(\mathrm{n}=59)$ of these patients were alive at time of inclusion and only five patients were younger than 65 years. Since the time of diagnosis, 182 patients had died. We further excluded 183 patients, of which approximately half was due to 


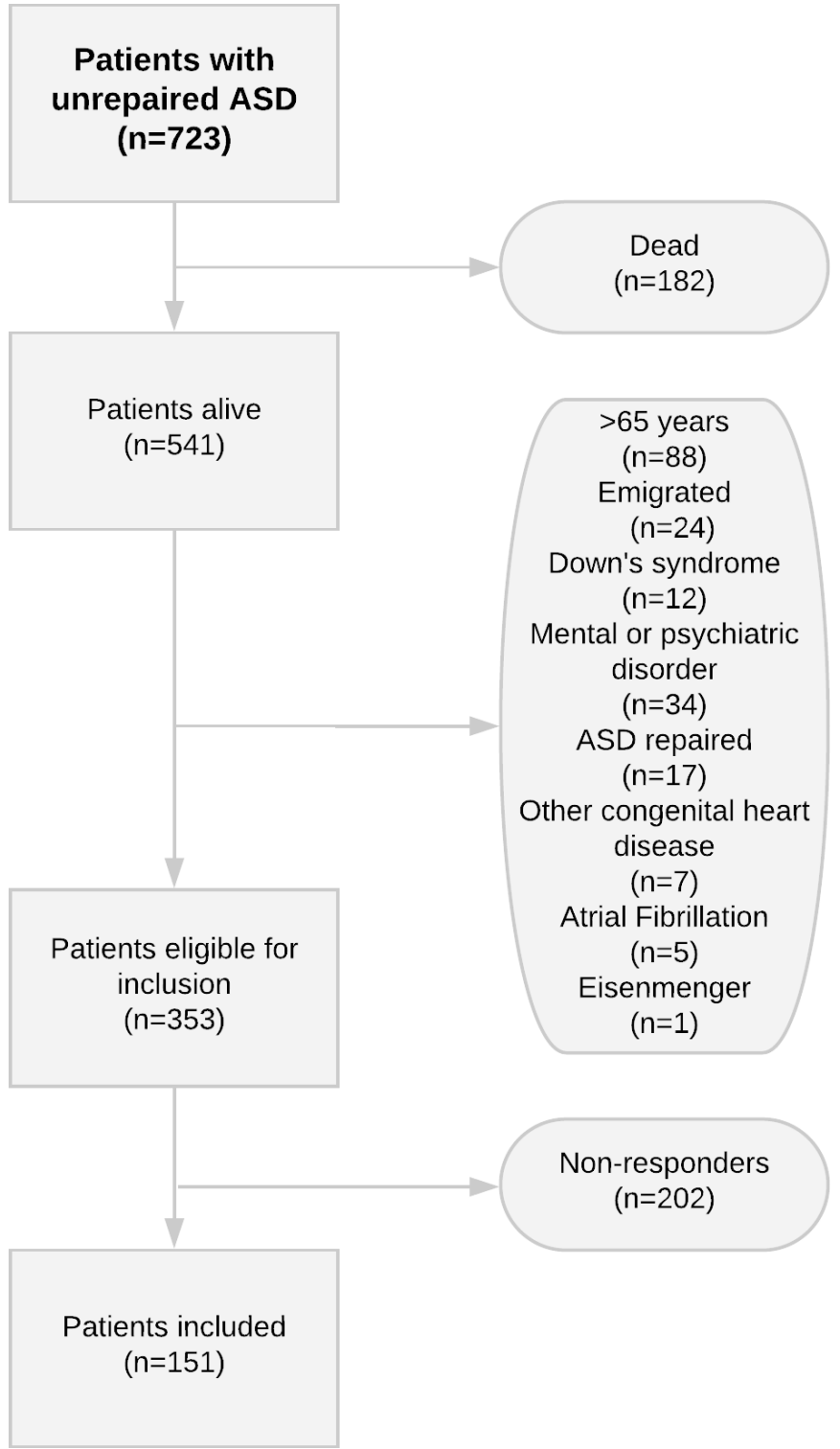

Figure 1 Flowchart of inclusion. ASD, atrial septal defects.

old age $(n=88)$, and 202 patients did not respond to or declined our invitation.

Consequently, we included 151 patients with ASD (mean age: 31.9 years) with a gender distribution of $61.6 \%$ female (figure 1). All patients characterised themselves as asymptomatic at time of inclusion, with no signs of arrhythmias. Patient characteristics are summarised in table 1 .

One patient previously underwent catheter ablation for Wolff-Parkinson-White Syndrome. Another patient had been diagnosed with symptomatic paroxysmal regular SVT during follow-up. When comparing included patients with ASD that attended for assessment and those patients who did not respond to the invitation letter, no differences in age, time of diagnosis or comorbidities were found.

\section{Resting ECG}

Twelve-lead electrocardiographic data are shown in table 2 .
Sinus rhythm was present in all patients. The QRS duration and PR-interval were within normal range. The percentage of patients with ASD with PR-interval $>200 \mathrm{~ms}$ was significantly higher in patients with SVT. P-wave duration and P-wave height were normal.

\section{Holter-recording}

The majority $(96 \%)$ completed all 7 days recording (table 3).

Most patients with small, unrepaired ASD had normal heart rhythm and patients daily heart rate total was within normal range. The most common arrhythmia was SVT $(n=24,16 \%)$ with the majority being non-sustained atrial arrhythmias (see online supplementary files 1 and 2), of which especially focal atrial tachycardia (FAT) was predominant $(n=21)$. The longest run was 26 premature complexes. Two patients had AF (20 year and 55 year). One patient was classified as having SVT of re-entry type. Furthermore, a considerable number of patients had NSVT $(n=12,8 \%)$. Two patients had ventricular runs of 10 beats or longer. All atrial and ventricular tachyarrhythmias were paroxysmal. Three patients had both SVT and NSVT. Patients with atrial or ventricular tachyarrhythmias were significantly older than patients without tachyarrhythmias and their age at time of diagnosis was significantly higher. No gender-based differences were present.

\section{Echocardiography}

Echocardiographic data are presented in table 4.

Echocardiographic outcome in all included patient. TR values were only measurable in 83 patients. $P$ values express the comparison between patients with ASD with tachyarrhythmias and patients with ASD without tachyarrhythmias.

The majority of defects had spontaneously closed since the time of diagnosis. Only 30 patients $(19.8 \%)$ (mean age: 36 year) still had an open ASD. One-third $(n=10)$ of patients with open defects had indication for defect closure due to dilated right heart chambers (mean age: 37 year). No right or left ventricular systolic or diastolic impairment was demonstrable in the entire cohort.

Patients with ASD and SVT or NSVT had a significantly higher right ventricular to left ventricular (RV/ LV) diastolic area ratio than patients with ASD without tachyarrhythmias. Pulmonary artery pressure estimated by the tricuspid regurgitation gradient tended to be higher in patients with SVT; however, the difference was not significant.

\section{6 min walk test}

The results of submaximal exercise capacity testing are shown in online supplementary table 5. One patient had recent hip surgery and was excluded from the test. There was no difference in walking distance, heart rate or saturation between patients with or without tachyarrhythmias. 
Table 1 Patients characteristics

\begin{tabular}{|c|c|c|c|c|c|c|}
\hline & $\begin{array}{l}\text { Total small } \\
\text { ASD }(n=151)\end{array}$ & $\begin{array}{l}\text { No tachyarrhythmia } \\
(n=118)\end{array}$ & $\begin{array}{l}\text { Supraventricular } \\
\text { tachycardia }(n=24)\end{array}$ & $P$ value & $\begin{array}{l}\text { Ventricular } \\
\text { tachycardia }(n=12)\end{array}$ & $P$ value \\
\hline Age (years) & $31.9 \pm 12.5$ & $28.2 \pm 9.3$ & $45.9 \pm 13.6$ & 0.001 & $45 \pm 14.2$ & 0.001 \\
\hline Height (cm) & $172 \pm 10$ & $171 \pm 11$ & $174 \pm 8$ & 0.289 & $170 \pm 8$ & 0.764 \\
\hline Weight (kg) & $75 \pm 20$ & $75 \pm 20$ & $78 \pm 18$ & 0.460 & $69 \pm 16$ & 0.350 \\
\hline Current smoker (\%) & 17.2 & 18.6 & 12.5 & 0.570 & 8.3 & 0.692 \\
\hline $\begin{array}{l}\text { Diet, self-estimated if } \\
\text { healthy }(\%)\end{array}$ & 37.7 & 32.7 & 54.1 & 0.062 & 50 & 0.337 \\
\hline $\begin{array}{l}\text { Self-estimated physics } \\
\text { (good) (\%) }\end{array}$ & 22 & 22.2 & 20.8 & 1.000 & 25 & 0.731 \\
\hline Chronic lung disease, $\mathrm{n}$ & 4 & 4 & 0 & - & 0 & - \\
\hline Diabetes, $\mathrm{n}$ & 1 & 0 & 1 & - & 0 & - \\
\hline $\begin{array}{l}\text { Pulmonary heart disease, } \\
\mathrm{n}\end{array}$ & 1 & 1 & 0 & - & 0 & - \\
\hline Hypertension, $\mathrm{n}$ & 3 & 0 & 3 & - & 0 & - \\
\hline $\begin{array}{l}\text { Ischaemic heart disease, } \\
n\end{array}$ & 3 & 0 & 2 & - & 1 & - \\
\hline
\end{tabular}

$P$ values express the comparison between ASD patients with tachyarrhythmias and ASD patients without tachyarrhythmias. ASD, atrial septal defects; BMI, body mass index.

\section{DISCUSSION}

In this nationwide cohort study, we examined the hidden burden of atrial and ventricular arrhythmias in asymptomatic adult patients with a small, unrepaired ASD without a previous diagnosis of AF. Our study demonstrated that one in five patients had arrhythmia even though the majority of patients had spontaneous closure of their ASD. The presence of arrhythmia was associated with signs of adverse RV remodelling, and the likelihood increased with age.

\section{Atrial arrhythmias}

Until recently, patients with small ASDs were considered as healthy as the general population. This assumption has been called into question in recent studies, establishing an increased risk of AF, pneumonia and stroke in these patients and a higher mortality when compared with the general population. ${ }^{2}{ }^{4}$ As such, these patients cannot be considered to have a benign long-term outcome and should remain under regular medical review throughout their life. ${ }^{3}$ The present study was designed to examine

Table 2 Electrocardiography

\begin{tabular}{|c|c|c|c|c|c|c|}
\hline & $\begin{array}{l}\text { Total small } \\
\text { ASD }(n=151)\end{array}$ & $\begin{array}{l}\text { No tachyarrhythmia } \\
(n=118)(78 \%)\end{array}$ & $\begin{array}{l}\text { Supraventricular } \\
\text { tachycardia }(n=24)\end{array}$ & P value & $\begin{array}{l}\text { Ventricular } \\
\text { tachycardia }(n=12)\end{array}$ & $P$ value \\
\hline QRS duration, ms & $83 \pm 18$ & $82 \pm 18$ & $83 \pm 18$ & 0.868 & $81 \pm 18$ & 0.773 \\
\hline $\mathrm{PR}>200 \mathrm{~ms}$ & $9 \%$ & $7 \%$ & $21 \%$ & 0.045 & $8 \%$ & 0.594 \\
\hline $\mathrm{P}$ wave duration, $\mathrm{ms}$ & $83 \pm 20$ & $82 \pm 20$ & $85 \pm 25$ & 0.554 & $87 \pm 20$ & 0.451 \\
\hline
\end{tabular}

ASD, atrial septal defects. 


\begin{tabular}{|c|c|}
\hline & $\begin{array}{l}\text { Small, } \\
\text { unrepaired } \\
\text { ASD }(n=151)\end{array}$ \\
\hline Supraventricular tachycardia, $\mathrm{n}(\%)$ & $24(16)$ \\
\hline Focal atrial tachycardia & 21 \\
\hline SVT (re-entry type) & 1 \\
\hline Atrial fibrillation & 2 \\
\hline 3-10 complexes & 13 \\
\hline$>10$ complexes & 11 \\
\hline Symptomatic & 7 \\
\hline Premature atrial complexes, $\mathrm{n}(\%)$ & $131(87)$ \\
\hline$\approx 0.5 \%$ & 25 \\
\hline $1 \%-2 \%$ & 4 \\
\hline $3 \%-6 \%$ & 4 \\
\hline Non-sustained ventricular tachycardia, $\mathrm{n}(\%)$ & $12(8)$ \\
\hline 3-10 complexes & 10 \\
\hline$>10$ complexes & 2 \\
\hline Premature ventricular complex, $\mathrm{n}(\%)$ & $121(80)$ \\
\hline >2000 per day & 6 \\
\hline Bigeminy/Trigeminy & $21(14)$ \\
\hline Left bundle branch block, $n$ & 1 \\
\hline Right bundle branch block, $\mathrm{n}$ & 3 \\
\hline AV-block, n (\%) & $14(9)$ \\
\hline First-degree & $4(3)$ \\
\hline Second-degree type 1 & $7(5)$ \\
\hline Second-degree advanced & $3(2)$ \\
\hline Third-degree & 0 \\
\hline Pauses & $6(4)$ \\
\hline Heartbeats (day) & $109866 \pm 14008$ \\
\hline
\end{tabular}

ASD, atrial septal defects; AV, atrioventricular; SVT, supraventricular tachycardia; TAPSE, tricuspid annular plane systolic excursion.

the covert burden of arrhythmia, as this might identify those at risk of developing symptomatic arrhythmia in the future and might cast light on the underlying mechanisms of arrhythmia, prior to the confounding effects of sustained arrhythmia itself. Our data showed that despite the ASD having closed spontaneously in the majority, $16 \%$ of patients had asymptomatic atrial arrhythmias, a number comparable to a previous study, reporting atrial arrhythmias (although many symptomatic) in $18.9 \%$ of patients with large significant ASD. ${ }^{8}$ In healthy individuals aged between 18 and 65 years, the prevalence of SVT seen during a 24 hours Holter monitoring was substantially lower $(2.2 \%$ and $2.5 \%)$ than in our patients. ${ }^{9}{ }^{10}$

Non-sustained atrial arrhythmia, predominately FAT, was the most common type of arrhythmia in patients with small ASDs. This is in contrast to a study by Labombarda et al investigating atrial arrhythmias in patients with congenital heart disease, which found FAT to be the third most common atrial arrhythmia and intra-atrial re-entrant tachycardia as the most common in patients with simple congenital defects. ${ }^{11}$ However, they also included patients with documented sustained atrial tachyarrhythmias, whereas our patients were asymptomatic without any history of arrhythmias.

It was somewhat surprising that only two patients $(1.3 \%)$ had paroxysmal AF, given the known association between ASD and AF in unoperated large ASD and in older patients after closure and with small unoperated ASD. ${ }^{212} 13$ However, given that all but five patients with known AF were dead or above 65 years of age, this outcome is not entirely unexpected. ${ }^{13}$ While speculative, it might be that the high occurrence of non-sustained atrial arrhythmias we demonstrated is a precursor for development of AF at older age, since FAT can trigger other atrial arrhythmias. ${ }^{14}$ Supportive of this, Labombarda et al found that a total of $12.2 \%$ of patients with congenital heart disease and FAT or intra-atrial re-entrant tachycardia developed new-onset $\mathrm{AF} .^{11}$ In this regard, the proportion of presenting arrhythmias due to FAT declined with age, while the proportion of AF steadily increased.

The high prevalence of arrhythmias we found might be, in the majority of patients, a consequence of having had an ASD, rather than having a small ASD, given that most patients with arrhythmias had spontaneous closure of the ASD by echocardiography at the time of examination. Indeed, there was no difference in the prevalence of SVT and NSVT between patients with a small, open ASD and patients with a spontaneously closed ASD. It is again speculative, but it might be that structural myocardial changes induced by volume overload occurring in early life are irreversible and provide the substrate for later arrhythmia. This is supported by our finding of increased $\mathrm{RV} / \mathrm{LV}$ ratio in patients with arrhythmia, no matter whether there was residual shunting, suggesting that myocardial dysfunction may both be persistent despite a lack of haemodynamic residua and is a driver of subsequent arrhythmia. Furthermore, closure of ASD does not abolish the risk of later atrial arrhythmia. Indeed, 21\% of Danish patients with ASD closure before the age of 25 developed AF during long-term follow-up, ${ }^{4}$ and patients diagnosed with small, unrepaired ASD have an increased risk of AF and a high prevalence of SVT and NSVT, despite $80 \%$ of them spontaneously close and spontaneously closure appearing within the first 5 years of life. ${ }^{45}$ This challenges the hypothesis that defect closure in young adulthood is protective against future arrhythmias, although clearly may modify their incidence and the other morbidity associated with chronic left-to right shunting. Further prospective studies are, however, needed to confirm these speculations.

\section{Ventricular arrhythmias}

Increased prevalence of ventricular arrhythmias has not been described in patients with small ASD. ${ }^{16}$ We found that NSVT was present in $8 \%$ of patients diagnosed with small ASD, considerably higher than the reported 
Table 4 Echocardiography

\begin{tabular}{|c|c|c|c|c|c|c|}
\hline & $\begin{array}{l}\text { Total small } \\
\text { ASD }(n=151)\end{array}$ & $\begin{array}{l}\text { No tachyarrhythmia } \\
(n=118)\end{array}$ & $\begin{array}{l}\text { Supraventricular } \\
\text { tachycardia }(n=24)\end{array}$ & $P$ value & $\begin{array}{l}\text { Ventricular } \\
\text { tachycardia }(n=12)\end{array}$ & $P$ value \\
\hline Open ASD, n (\%) & $30(20)$ & $23(19)$ & $4(16)$ & 1.000 & $3(23)$ & 1.000 \\
\hline Indication for defect closure & $10(6.6)$ & $6(5)$ & $3(13)$ & 0.178 & $1(8)$ & 0.501 \\
\hline
\end{tabular}

(dilatation of right heart

chambers), n (\%)

\begin{tabular}{lllllll} 
Septal aneurisms, $\mathrm{n}(\%)$ & $18(12)$ & $15(13)$ & $2(8)$ & 1.000 & $1(8)$ & 1.000 \\
Ejection fraction (\%) & $62.4 \pm 3.9$ & $62.3 \pm 3.3$ & $63.1 \pm 6.2$ & 1.000 & $62.8 \pm 3.3$ & $30.3 \pm 5.8$ \\
\hline LV area $\left(\mathrm{cm}^{2}\right)$ & $31.3 \pm 5.9$ & $31.4 \pm 6.4$ & $31.4 \pm 3.2$ & 0.978 & 1.000 & 0.559 \\
LA area $\left(\mathrm{cm}^{2}\right)$ & $16.4 \pm 4.1$ & $16.1 \pm 4.0$ & $17.7 \pm 4.8$ & 0.100 & $15.9 \pm 3.5$ \\
RV area $\left(\mathrm{cm}^{2}\right)$ & $15.8 \pm 6.1$ & $15.5 \pm 5.3$ & $17.0 \pm 9.5$ & 0.320 & $15.3 \pm 5.4$ & $12.9 \pm 3.5$ \\
RA area $\left(\mathrm{cm}^{2}\right)$ & $12.5 \pm 3.3$ & $12.3 \pm 3.3$ & $13.3 \pm 3.2$ & 0.196 & 0.853 \\
RV/LV diastolic area, ratio & $0.7 \pm 0.09$ & $0.68 \pm 0.08$ & $0.72 \pm 0.07$ & 0.038 & $0.75 \pm 0.12$ & 0.650 \\
TR (mm Hg) & $16 \pm 5.2$ & $15.8 \pm 5.3$ & $18.7 \pm 4.5$ & 0.070 & 0.005 \\
RV fractional area change $(\%)$ & $51.9 \pm 8.6$ & $51.4 \pm 9.3$ & $53.2 \pm 4.8$ & 0.372 & $52.8 \pm 7.5$ \\
TAPSE (mm) & $24.5 \pm 3.8$ & $24.2 \pm 3.9$ & $25.8 \pm 2.5$ & 0.078 & $24.6 \pm 4.8$ \\
TR (mm Hg) & $16 \pm 5.3$ & $15.8 \pm 5.3$ & $18.7 \pm 4.5$ & 0.079 & $15.8 \pm 6.1$ \\
VCl diameter expiration/ & $3.5 \pm 1.6$ & $3.4 \pm 1.5$ & $3.4 \pm 1.2$ & 0.985 & $3.9 \pm 2.4$ \\
inspiration (ratio) & & & & 0.690 \\
\hline
\end{tabular}

ASD, atrial septal defects; LA, left atrium; LV, left ventricle; RA, right atrium; RV, right ventricle; TR, tricuspid regurgitation; VCI, vena cava inferior.

prevalence of NSVT in healthy adults $(0.7 \%$ and $2 \%)$ on 24 hours Holter recordings. ${ }^{9}{ }^{10}$ That said, the prevalence of NSVT in congenital heart disease is largely unknown. However, in a study of patients with congenital heart disease presenting either at the emergency room or at the outpatient clinic with a ventricular tachyarrhythmia, $71 \%$ of admissions were due to NSVT. ${ }^{17}$ Surprisingly for the authors of that paper, $7 \%$ of their patients had a diagnosis of ASD (mean age of 48 year at event presentation). Reassuringly, patients admitted with NSVT rarely developed secondary sustained ventricular tachycardia or ventricular fibrillation during a 5 -year follow-up period. ${ }^{17}$ Additionally, despite NSVT occurring more frequently in sudden cardiac death cases, NSVT was not associated with sudden cardiac death in patients with congenital heart disease. ${ }^{18}$ There are, however, reports of sudden cardiac death in patients with both repaired and small unrepaired ASD. ${ }^{18}$ Additionally, sudden unexpected death was found to be the most common cause of death $(40 \%)$ in patients with simple congenital heart disease, and the authors speculate that these events are likely to have an underlying arrhythmic cause.${ }^{19}$ Patients with familial ASD are also recommended to be screened for gene mutation NKX2-5, which carries an increased risk for early sudden death. ${ }^{20}$ We did not genotype our patients, but future mechanistic studies might include screening for potential culprit genes that predispose to these adverse outcomes in patients with ASD.

\section{Clinical perspective}

The ACC/AHA 2008 ${ }^{21}$ guidelines suggest routine follow-up of patients with a small ASD without evidence of right ventricular enlargement or pulmonary arterial hypertension every 2-3 years. $^{21}$ Clinical examination should include repeat echocardiogram and assessment of symptoms, especially arrhythmias, since the presence of atrial arrhythmia more than doubled the risk of congestive heart failure in adult patients with congenital heart disease. ${ }^{8}$ This correlates with our earlier data, demonstrating that the most frequent cause of death in patients with small ASD is non-ischaemic heart failure. ${ }^{2}$ Therefore, considering the high prevalence of arrhythmias and the natural history of small ASDs, where potential progression in haemodynamic significance of the ASD can occur, we support a continuous follow-up for these patients. Since patients with a small ASD and SVT or NSVT were significantly older and diagnosed later than ASD patients without arrhythmias, traditional risk factors for arrhythmias should be addressed as well. The role of sequential Holter-recording in asymptomatic patients remains to be determined and the clinical utility of this approach should be examined in large cohort longitudinal studies.

\section{Limitation}

We used transthoracic echocardiogram to assess the presence and characteristics of the ASD. Transoesophageal echocardiography with a bubble study ideally should have been used to minimise the risk of overlooking small defects and persistent foramen ovale. However, we found that this was not practically or ethically feasible and discovery of tiny defects would not influence the overall interpretation of our data.

Unfortunately, information on defect characteristics and shunt dimensions at time of diagnosis was not 
available. Therefore, we had to rely on the clinical evaluation at that time to classify the defect as 'small' and insignificant. Nevertheless, patients eligible for inclusion were according to our data representative of the patients seen in adult congenital clinics today. Furthermore, the included patients were representative for the entire cohort eligible for inclusion. No differences in characteristics were found between the patients included and patients who did not respond to our invitation letter. Three patients had both SVT and NSVT and were featured in both groups. However, neither patient characteristics nor echocardiographic data differed when compared with patients with only SVT or NSVT.

Given all included patients said they were asymptomatic at time of inclusion, it is perhaps surprising that seven patients reported symptoms during their Holter recording. However, patients might not have been aware of or neglected such cardiac symptoms before being specifically instructed to notice them as a part of the examination.

Finally, the prevalence of SVT and NSVT found in our patients was obtained during a 48-hour Holter-recording, but due to the lack of normative 48 hours material in healthy individuals, our results were compared with findings as seen in a 24-hour Holter-recording. When increasing the monitoring window from 1 day to 2 days, a slightly higher prevalence of arrhythmias is to be expected in healthy adults. However, the higher prevalence of hidden arrhythmias reported in our patients when compared with the 24-hour Holter-recording reference populations cannot be entirely contributed to prolonged monitoring.

\section{CONCLUSION}

In this nationwide study, patients diagnosed with a small, unrepaired ASD without a known diagnosis of AF had a hidden burden of both atrial and ventricular tachyarrhythmias, predominately non-sustained atrial arrhythmia and NSVT. Arrhythmias were present despite spontaneous closure of the ASD and were associated with increased RV/ $\mathrm{LV}$ area ratio, suggesting that associated changes in atrial and ventricular myocardium are partly irreversible. These results support the guidelines for management of adults with small ASD, recommending continued follow-up with a clinical focus on arrhythmias.

Acknowledgements We gratefully acknowledge research secretary Jette Breiner, research nurse Vibeke Laursen and echo-technician Bente Mortensen for their highly valuable contributions.

Contributors SU, CN, JEN-K, VEH: construction of idea, planning of research, conduct of research, manuscript writing and revision. AR, JCN: idea inception and formulation, data interpretation, writing of the manuscript.

Funding This work was supported by Aarhus University and Karen Elise Jensen's Foundation.

Competing interests None declared.

Patient consent for publication Not required.
Provenance and peer review Not commissioned; externally peer reviewed.

Data availability statement No additional data are available.

Open access This is an open access article distributed in accordance with the Creative Commons Attribution Non Commercial (CC BY-NC 4.0) license, which permits others to distribute, remix, adapt, build upon this work non-commercially, and license their derivative works on different terms, provided the original work is properly cited, appropriate credit is given, any changes made indicated, and the use is non-commercial. See: http://creativecommons.org/licenses/by-nc/4.0/.

\section{REFERENCES}

1. Vecht JA, Saso S, Rao C, et al. Atrial septal defect closure is associated with a reduced prevalence of atrial tachyarrhythmia in the short to medium term: a systematic review and meta-analysis. Heart 2010;96:1789-97.

2. Nyboe C, Karunanithi Z, Nielsen-Kudsk JE, et al. Long-term mortality in patients with atrial septal defect: a nationwide cohort-study. Eur Heart J 2018;39:993-8.

3. Udholm S, Nyboe C, Karunanithi Z, et al. Lifelong burden of small unrepaired atrial septal defect: results from the Danish national patient registry. Int J Cardiol 2019;283:101-6.

4. Nyboe C, Olsen MS, Nielsen-Kudsk JE, et al. Atrial fibrillation and stroke in adult patients with atrial septal defect and the long-term effect of closure. Heart 2015;101:706-11.

5. Nyboe C, Olsen MS, Nielsen-Kudsk JE, et al. Risk of pneumonia in adults with closed versus unclosed atrial septal defect (from a nationwide cohort study). Am J Cardiol 2014;114:105-10.

6. Lynge E, Sandegaard JL, Rebolj M. The Danish national patient register. Scand J Public Health 2011;39(7_suppl):30-3.

7. Schmidt M, Schmidt SAJ, Sandegaard JL, et al. The Danish national patient Registry: a review of content, data quality, and research potential. CLEP 2015;7:449-90.

8. Bouchardy J, Therrien J, Pilote L, et al. Atrial arrhythmias in adults with congenital heart disease. Circulation 2009;120:1679-86.

9. Hingorani P, Karnad DR, Rohekar P, et al. Arrhythmias seen in baseline 24-hour Holter ECG recordings in healthy normal volunteers during phase 1 clinical trials. J Clin Pharmacol 2015;2016:885-93.

10. Stinson JC, Pears JS, Williams AJ, et al. Use of $24 \mathrm{~h}$ ambulatory ECG recordings in the assessment of new chemical entities in healthy volunteers. Br J Clin Pharmacol 1995;39:651-6.

11. Labombarda F, Hamilton R, Shohoudi A, et al. Increasing prevalence of atrial fibrillation and permanent atrial arrhythmias in congenital heart disease. J Am Coll Cardiol 2017;70:857-65.

12. Karunanithi Z, Nyboe C, Hjortdal VE. Long-term risk of atrial fibrillation and stroke in patients with atrial septal defect diagnosed in childhood. Am J Cardiol 2017;119:461-5.

13. Gatzoulis MA, Freeman MA, Siu SC, et al. Atrial arrhythmia after surgical closure of atrial septal defects in adults. $N$ Engl J Med 1999;340:839-46.

14. Rosso R, Kistler PM. Focal atrial tachycardia. Heart 2010;96:181-5.

15. Garne E. Atrial and ventricular septal defects - epidemiology and spontaneous closure. J Matern Fetal Neonatal Med 2006;19:271-6.

16. Khairy P, Van Hare GF, Balaji S, et al. PACES/HRS expert consensus statement on the recognition and management of arrhythmias in adult congenital heart disease. Can J Cardiol 2014;30:e1-63.

17. Teuwen CP, Ramdjan TTTK, Götte M, et al. Non-sustained ventricular tachycardia in patients with congenital heart disease: an important sign? Int J Cardiol 2016;206:158-63.

18. Koyak Z, Harris L, de Groot JR, et al. Sudden cardiac death in adult congenital heart disease. Circulation 2012;126:1944-54.

19. Videbæk J, Laursen HB, Olsen M, et al. Long-term nationwide follow-up study of simple congenital heart disease diagnosed in otherwise healthy children. Circulation 2016;133:474-83.

20. Ellesøe SG, Johansen MM, Bjerre JV, et al. Familial Atrial Septal Defect and Sudden Cardiac Death: Identification of a Novel NKX25 Mutation and a Review of the Literature. Congenit Heart Dis 2016;11:283-90.

21. Warnes CA, Williams RG, Bashore TM, et al. ACC/AHA 2008 guidelines for the management of adults with congenital heart disease: Executive summary - A report of the American College of Cardiology/American Heart Association Task Force on practice guidelines. Circulation 2008;118:2395-451. 